\title{
CLOSED SETS WITHOUT MEASURABLE MATCHING
}

\author{
M. LACZKOVICH
}

(Communicated by R. Daniel Mauldin)

\begin{abstract}
We construct a rectangle in the unit square such that its perimeter contains a matching (i.e. the graph of a bijection of the unit interval onto itself) but does not contain a Borel matching or a matching measurable with respect to the linear measure.
\end{abstract}

Let $I$ and $I^{2}$ denote the unit interval and the unit square, respectively. A subset of $I^{2}$ is called a matching if it is the graph of a one-to-one map of $I$ onto itself. By a theorem of D. König [5], if each section $K_{x}=\{y:(x, y) \in K\}(x \in I)$ and $K^{y}=\{x:(x, y) \in K\}(y \in I)$ of the set $K \subset I^{2}$ consists of exactly $n$ elements $(n$ is finite), then $K$ contains a matching. For infinite $n$ this was proved by J. Kaniewski and C. A. Rogers [4].

It may happen that a Borel subset of $I^{2}$ contains a matching but does not contain a Borel matching or even a matching measurable with respect to the linear measure. Borel sets with this property were constructed by Kaniewski and Rogers [4] and Mauldin [6] (see also [2, §4]). The set given in [4] has countably infinite sections; the sections of Mauldin's set are uncountable. In this note we present a closed set with the property above. It is the union of four segments and has finite sections.

THEOREM. Let $u \in I$ be an irrational number and let $R$ denote the perimeter of the rectangle with vertices $A_{0}(1,1-u), A_{1}(1-u, 1), A_{2}(0, u)$, and $A_{3}(u, 0)$. Then $R$ contains a matching but does not contain a Borel matching. Moreover, no matching in $R$ is measurable with respect to the linear (Hausdorff) measure.

PROOF. By symmetry, we may assume $0<u<1 / 2$. The set $R$ defines a bipartite graph $G$ as follows. Let $I_{1}$ and $I_{2}$ be two copies of $I$, and let the points $x \in I_{1}$ and $y \in I_{2}$ be connected by an edge if and only if $(x, y) \in R$. Then $R$ contains a matching if and only if $G$ contains a 1 -factor. Also, $G$ contains a 1 -factor if and only if each connected component of $G$ contains a 1 -factor. Since every point of $G$ has degree 1 or 2 , the connected components of $G$ are paths and circuits. (As for the notions of graph theory used above, see [1].) The circuits, infinite paths and finite paths of even length contain 1-factors, and hence, in order to find a 1-factor in $G$ it is enough to prove that no component of $G$ is a finite path of odd length.

Suppose this is not true and let $C=\left\{x_{1}, x_{2}, \ldots, x_{n}\right\}$ be a component of $G$, where $\left(x_{i-1}, x_{i}\right) \in R$ for every $i=2, \ldots, n$, and $n$ is odd. Then $x_{1}$ and $x_{n}$ both belong to $I_{1}$ or $I_{2}$ and have degree 1 . Since the only elements of degree 1 in $G$ are 0 and 1 , this implies that $x_{1}=0, x_{n}=1$ or $x_{1}=1, x_{n}=0$.

Received by the editors July $1,1987$.

1980 Mathematics Subject Classification (1985 Revision). Primary 28A05; Secondary 05C70. 


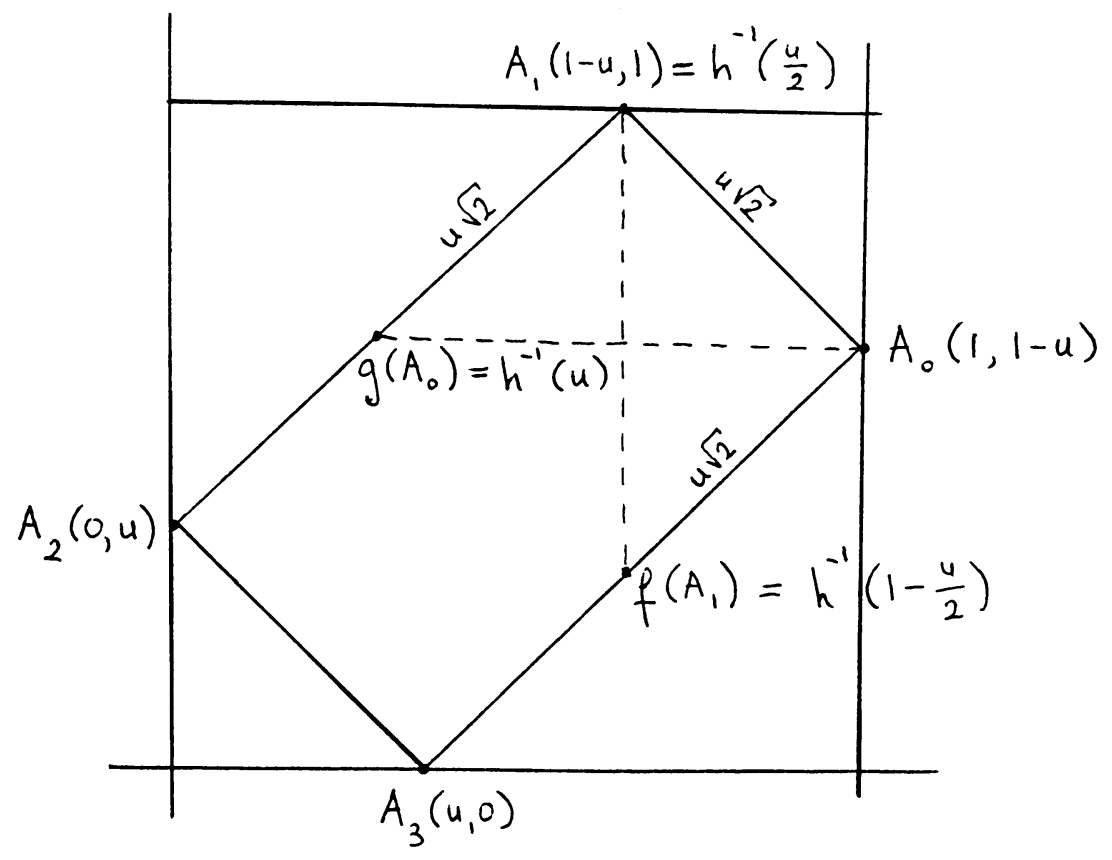

FIGURE 1

If $(x, y) \in R$ and $(x, y)$ belongs to the segment joining $A_{0}$ and $A_{1}$ then $y=$ $-x+2-u$. If $(x, y)$ belongs to the other three segments then $y= \pm x \pm u$. This implies, by induction on $k$, that $x_{k}= \pm x_{1}+2 a_{k}+b_{k} u(k=2, \ldots, n)$, where $a_{k}$ and $b_{k}$ are integers. In particular, $x_{n}= \pm x_{1}+2 a_{n}+b_{n} u$, and hence $b_{n} u=x_{n} \pm x_{1}-2 a_{n}=$ $\pm 1-2 a_{n}$. Thus $b_{n} u$ is an odd integer which implies $b_{n} \neq 0$. Therefore $u$ is rational, which contradicts our assumption. Hence $G$ does not contain components of odd length and, consequently, contains a matching.

Let $\mu$ denote the normalized linear measure on $R$, i.e. let $\mu(H)=\lambda_{1}(H) / 2 \sqrt{2}$ for every measurable $H \subset R$. We define two maps, $f$ and $g$, of $R$ into itself as follows. We put $f\left(A_{0}\right)=A_{0}$ and $f\left(A_{2}\right)=A_{2}$. If $(x, y) \in R$ and $x \neq 0,1$ then there is a unique $z \neq y$ such that $(x, z) \in R$, and we define $f(x, y)=(x, z)$. Also, we define $g\left(A_{1}\right)=A_{1}$ and $g\left(A_{3}\right)=A_{3}$. If $(x, y) \in R$ and $y \neq 0,1$ then there is a $z \neq x$ such that $(z, y) \in R$ and we put $g(x, y)=(z, y)$. It is easy to check that $f$ and $g$ are measure-preserving homeomorphisms of $R$ onto itself with $f^{-1}=f$ and $g^{-1}=g$.

We prove that $g \circ f$ is ergodic on $R$, that is, if $H$ is measurable and $g \circ f(H)=H$ then $\mu(H)=0$ or $\mu(H)=1$.

Let $T$ denote the circle group with the Lebesgue measure, and let $h: R \rightarrow T$ be a measure-preserving homeomorphism of $R$ onto $T$ such that $h\left(A_{0}\right)=0, h\left(A_{1}\right)=$ $u / 2, h\left(A_{2}\right)=1 / 2$, and $h\left(A_{3}\right)=(1+u) / 2$. Let $k=h \circ g \circ f \circ h^{-1}$, then $k$ is a measure-preserving homeomorphism of $T$ onto itself. Therefore either $k(t)=t+c$ or $k(t)=-t+c(t \in T)$ with a constant $c \in T$. It is easy to check that $k(0)=u$ and $k(1-(u / 2))=u / 2$ (see Figure 1). Hence $k(t)=t+u$ for every $t \in T$. Since $u$ is irrational, $k$ is ergodic on $T$ (see [3, p. 26]), and hence $g \circ f=h^{-1} \circ k \circ h$ is ergodic on $R$. (This argument is similar to that in $[8$, p. 10].) 
Now suppose that $H$ is a measurable matching in $R$. Then $H \cap f(H)$ is finite and $H \cup f(H)=R$. This implies $\mu(H)=1 / 2$. Also, $H \cap g(H)$ is finite and $H \cup g(H)=R$, and hence the symmetric difference of the sets $H$ and $g \circ f(H)$ is finite. Since $g \circ f$ is ergodic, this gives $\mu(H)=0$ or $\mu(H)=1$. This contradiction completes the proof.

REMARK. It was proved by R. Rado in [7] that if the sections of a set $K \subset I^{2}$ are finite then $K$ contains a matching if and only if $\operatorname{card} \bigcup\left\{K_{x}: x \in H\right\} \geq \operatorname{card} H$ and card $\bigcup\left\{K^{y}: y \in H\right\} \geq \operatorname{card} H$ hold for every finite set $H \subset I$.

Our theorem shows that Rado's condition does not ensure the existence of a Borel matching even if $K$ is compact. The same is true for König's condition. Indeed, let $R$ be a rectangle as in the theorem above, and let $K$ be the union of $R$ and the points $(0,0)$ and $(1,1)$. Then $K$ is compact, $\operatorname{card} K_{x}=\operatorname{card} K^{y}=2$ for every $x, y \in I$ and $K$ does not contain a measurable matching.

\section{REFERENCES}

1. B. Bollobas, Graph theory, Graduate Texts in Math., vol. 63, Springer-Verlag, Berlin and New York, 1979.

2. S. Graf and R. D. Mauldin, Measurable one-to-one selections and transition kernels, Amer. J. Math. 107 (1985), 407-426.

3. P. R. Halmos, Lectures on ergodic theory, Chelsea, 1956.

4. J. Kaniewski and C. A. Rogers, Double uniformization, J. London Math. Soc. (2) 22 (1980), 521-533.

5. D. König, Sur les correspondences multivoques des ensembles, Fund. Math. 8 (1926), 114-134.

6. R. D. Mauldin, One-to-one selections-marriage theorems, Amer. J. Math. 104 (1982), 823-828.

7. R. Rado, Factorization of even graphs, Quart. J. Math. Oxford Ser. 20 (1949), 95-104.

8. V. N. Sudakov, On independent complementation to two partitions in the case when there exists a bounded density, Proc. Steklov Inst. Math. 111 (1970), 3-11 (Russian).

DEPARTMENT OF ANALYSIS, EÖTVÖS LORÁND UNIVERSITY, BUDAPEST, HUNGARY 